\title{
AMLODIPINE RELATED GINGIVAL OVERGROWTH IN A PATIENT WITH ASOCIATED AUTOSOMAL POLYCYSTIC KIDNEY DISEASE
}

\author{
Fidan Bahtiar Ismail ${ }^{1}$, Horia Traian Dumitriu ${ }^{1}$, Anca Silvia Dumitriu ${ }^{1}$, Gener Ismail ${ }^{2}$ \\ ${ }^{1}$ Department of Periodontology, "Carol Davila" University of Medicine and Pharmacy, Bucharest \\ ${ }^{2}$ Department of Nephrology, Urology, Transplant Immunology, Dermatology and Allergology, \\ "Carol Davila" University of Medicine and Pharmacy, Bucharest
}

\begin{abstract}
Background. The paper describes the case of a 62 years non-smoking male patient who was referred to the Department of Periodontology, "Carol Davila" University of Medicine and Pharmacy, complaining of gingival overgrowth. The patient's medical history revealed that he suffers from autosomal dominant polycystic kidney disease chronic renal failure and hypertension; the patient was under treatment with amlodipine, a calcium channel blocker for 18 months.

Case presentation. The patient underwent cause-related periodontal therapy and flap surgery in areas where only partial resolution of the gingival overgrowth was obtained after completion of initial phase therapy; amlodipine was not substituted because of medical reasons.

Results. We obtained complete resolution of the gingival enlargement, even without any change in the causative drug regimen; the patient was placed in a maintenance program, with regular appointments at 3 months; he successfully managed to maintain periodontal and oral health.

Conclusions. If possible, all patients who are about to receive calcium channel blockers, should have a detailed periodontal assessment performed and if any periodontal disease discovered, treated properly. In case of established gingival overgrowth, non-surgical and/or surgical periodontal therapy could induce partial or complete remission of enlargement. Change of medication is also an option.
\end{abstract}

Keywords: gingival overgrowth, amlodipine, autosomal dominant polycystic kidney disease (ADPKD), scaling and root planning, gingivectomy

\section{INTRODUCTION}

Autosomal dominant polycystic kidney disease (ADPKD) is the most common of the congenital renal cystic disorders, a group of diseases with related but distinct pathogenesis, described by the development of renal cysts and multiple extra-renal manifestations. Hypertension occurs in nearly $50-70 \%$ of the cases even before any major reduction in glomerular filtration rate appears (1). Patients with ADPKD and hypertension respond well to angiotensin converting enzyme (ACE) inhibitors and to angiotensin II- receptor blockers (ARBs), the goal being a blood pressure of maximum 130/80 $\mathrm{mmHg}$. In patients who develop a clinically significant decline in renal function, calcium channel blockers might be considered (2).

Gingival overgrowth is now a recognized unwanted side-effect associated with many of calcium channel blockers. Of this large group of drugs, the dihydropyridines are the agents most frequently implicated (3). In 1994, Seymour et al. (3) were also the first who reported that amlodipine, a third generation dihydropyridine, used for treatment of hypertension and angina, was causing gingival overgrowth as side effect. 


\section{CASE PRESENTATION}

\section{Patient evaluation}

A 62 years non-smoking male patient was referred to the Department of Periodontology for periodontal assessment with chief complaints of gingival overgrowth and tooth mobility. He was unable to provide detailed information about when tooth mobility started but he could appreciate that the debut of gingival enlargement was approximately 10 months prior to his visit.

The medical history revealed that patient suffered from ADPKD accompanied by chronic renal failureandseverehypertension for 2 years and was under treatment with diuretics (furosemide), angiotensine converting enzyme inhibitors (enalapril, 20 $\mathrm{mg} /$ day) central adrenergic agents, acetylsalicylic acid $75 \mathrm{mg}$ /day and calcium channel blockers (amlodipine besylate, $10 \mathrm{mg}$ /day). The patient has been taking amlodipine for 18 months.

Intraoral examination disclosed a dentate patient with a few missing teeth $(18,17,28,36,41,46)$ and no coronal restorations with the exception of 47 which presented an overhanging occlusal-distal amalgam restoration.

Generalized gingival overgrowth (GO) was present on both facial and oral surfaces with red, ulcerated and lobulated papillae, gingival margins and attached gingiva, less pronounced at the lower left sextant; purulent secretion was evident in the gingival sulcus. Patient's oral hygiene was poor with heavy generalized plaque and calculus deposits throughout dentition. (Fig. 1)

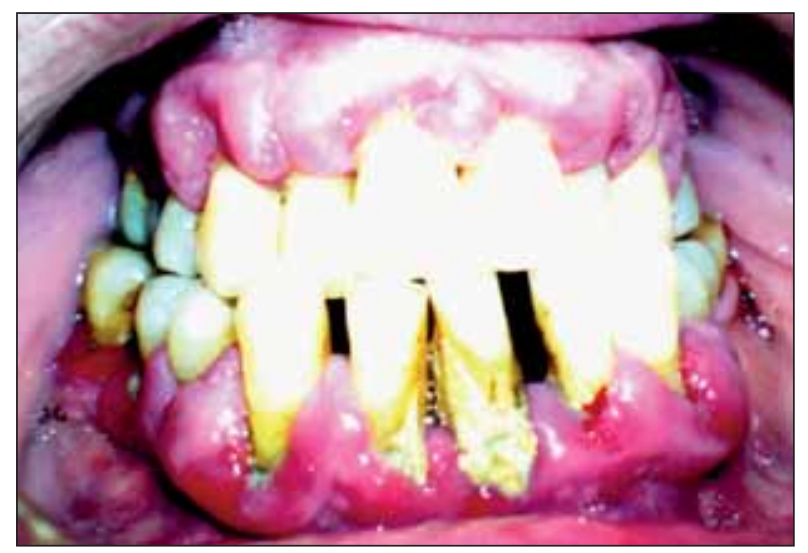

FIGURE 1. Generalized gingival overgrowth

Detailed clinical periodontal examination was performed with a manual University of North Carolina (UNC-15) probe and revealed: generalized bleeding on probing, generalized and significant periodontal pocketing, with a maximum depth of $8 \mathrm{~mm}$, class I furcation involvement at 37, class
II at 16, 26 and 27 and class III at 38 and 47, and pathologic class III tooth mobility on teeth 31 and 38.

Orthopantomographicexamination showed generalized horizontal bone loss with localized angular bone defects at teeth no. 44 and 33, furcation involvement in all molar teeth and periapical pathology in teeth no. 16 and 26, incorrect endodontic treatment on tooth no. 47 and presence of submerged vestibular roots of tooth no. 17. (Fig. 2)

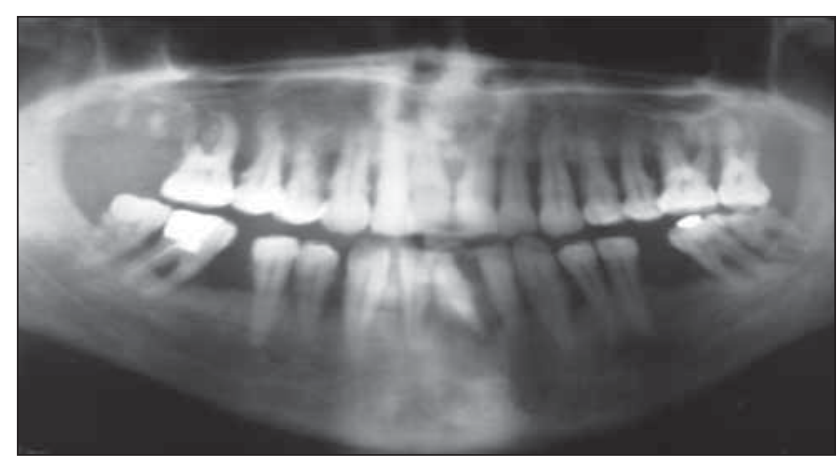

FIGURE 2. Initial radiological findings

The dental history of the patient revealed that the third upper right molar and the first mandibular molars were extracted because of caries complications long time ago, while the lower right first incisor was spontaneously lost 1 year prior to his dental examination.

Occlusal examination discovered a moderate class III malocclusion which might have been a contributor to the periodontal destruction through occlusal trauma.

The patient admitted that he had never undergone periodontal therapy such as scaling and root planning and no brushing instructions were given

The laboratory data showed normal white blood cell count, no anaemia, increased erythrocyte sedimentation rate $40 \mathrm{~mm} / 1 \mathrm{~h}$, normal liver enzymes, elevated creatinine level $1.9 \mathrm{mg} / \mathrm{dl}$ (eGFR $36 \mathrm{~mL} /$ $\min 1.73 \mathrm{~m}^{2}$ ). Serology for autoimmunity and infectious diseases was negative.

Based on the medical history, clinical, radiographic and paraclinical findings, the patient was diagnosed with amlodipine related gingival enlargement superimposed on a generalized severe chronic periodontitis.

\section{Treatment and evolution}

Periodontal treatment chief objectives were resolution and control of the plaque-induced gingival inflammation, stopping further progression of periodontal tissue destruction and preventing from recurrence of periodontal disease. 
Initial treatment phase aimed at eliminating and preventing the recurrence of local supra- and subgingival bacterial plaque deposits from the tooth surfaces and included the following procedures: oral hygiene instructions and improvement of selfperformed plaque control in association with chemical plaque control (mouthwashes with chlorhexidine $0.2 \%$ twice a day), supra- and subgingival scaling and root planning, removal of local plaque retentive factors such as overhanging restorations, endodontic treatment of teeth no $16,26,47$, extraction of compromised teeth (i.e. 31,38 ). Considering the medically compromised status of the patient, scaling and root planning were performed in conjunction with systemic antibiotics (amoxicillin $500 \mathrm{mg}$ tid and metronidazole $250 \mathrm{mg}$ tid for 7 days). The patient was also referred to nephrologist with the request of amlodipine substitution or withdrawal but given the general associated pathology none was achieved.

After the initial course of systemic antibiotherapy, the patient was placed, with agreement from the specialist, on a subantimicrobial dose of doxycycline hyclate $20 \mathrm{mg}$ twice a day, for 3 months as part of host response modulation treatment strategy.

At 1,2 and 3 months after initial therapy the patient was re-evaluated and additional surgical and corrective treatment was considered to be necessary in order to completely eliminate periodontal pockets and to restore masticatory and occlusal function. The patient presented a good plaque control with a Plaque Index of $10 \%$.

Surgical treatment consisted inexternal bevel gingivectomy combined with internal bevel gingivectomy, mucoperiostal flap, curettage and root planning in areas where only limited results (partial resolution of the gingival overgrowth) were obtained after initial therapy. During surgery, a piece of gingiva was collected and sent for histological examination. The histological findings are consequent with those encountered in any drug-influenced gingival overgrowth (Fig. 3).

Adjunctive corrective therapy consisted of:hemisection of 47 , maintaining both mesial and distal roots, extraction of 32 and 42 in order to facilitate prosthetic rehabilitation in the frontal area of the lower maxilla, endodontic treatment of the abutment teeth andprosthetic rehabilitation of the left and right lateral areas of the mandible.

The patient was placed on a regular follow-up program at every 3 months during the first year after completion of periodontal therapy.On each session, plaque and calculus indices were recorded

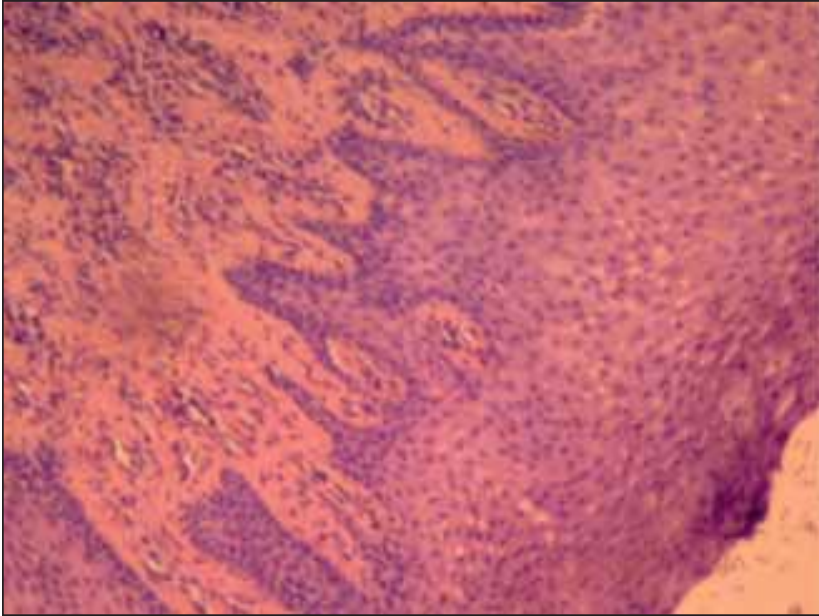

FIGURE 3. Histological aspect at the time of surgery

and when necessary, professional scaling, root planning and tooth polishing were performed. Oral hygiene instructions were reemphasized.

Clinical and radiographic reevaluation was performed 15 months after initial presentation and 12 months after surgical therapy (Fig. 4, Fig. 5). Also, blood investigations were performed at 11 month from initial presentation; negative $\mathrm{C}$ reactive protein and normal erythrocyte sedimentation rate were found.

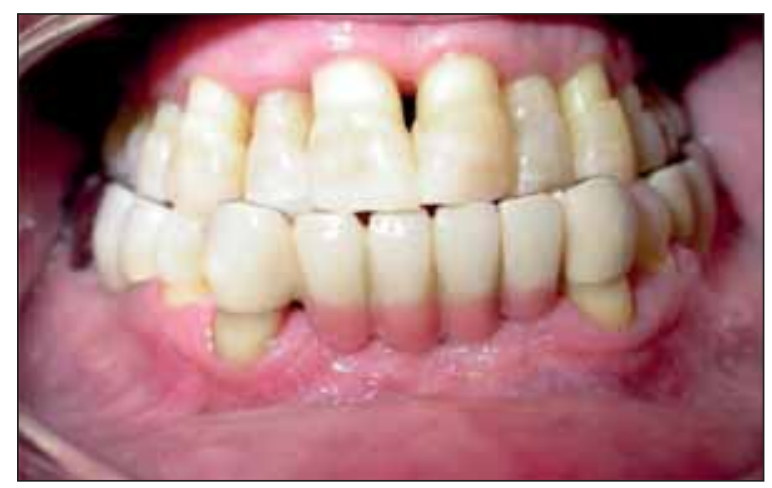

FIGURE 4. Clinical aspect 1 year after completion of active periodontal therapy

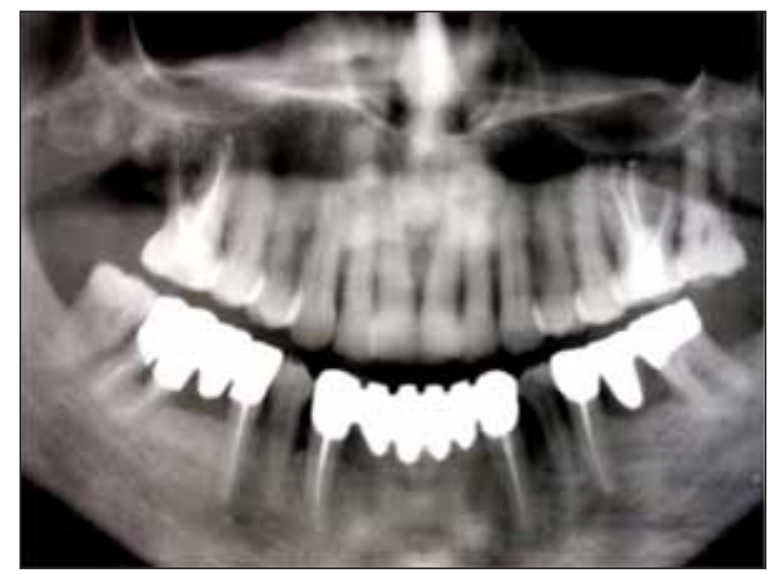

FIGURE 5. Radiological aspect 1 year after completion of active therapy 


\section{DISCUSSION}

American Academy of Periodontology included drug associated gingival overgrowth in the category of dental plaque-induced gingival diseases, gingival diseases modified by medication (4) because evidence suggests that pre-existing gingival inflammation is necessary for its occurrence and a good oral hygiene with effective plaque control might reduce the severity or even prevent from its development (5).

The prevalence of gingival overgrowth in patients taking amlodipine was reported to be 3.3\% (6), $1.7 \%$ in the study of Ellis et al. in 1999 (7) and $1.3 \%$ in the study conducted by Ono and co-workers in 2010 (8), all values being lower than the prevalence rate reported in patients taking nifedipine $47.8 \%$ (9). Clinical manifestation of gingival enlargement frequentlyappears within 1 to 3 months (1 to 9 months according to drug information paper) after initiation of treatment with amlodipine (10).

The pathogenesis of GO is still not fully understood and the treatment is mostly limited to the maintenance of a good oral hygiene, initial phase therapy and/or surgical removal of the hyperplastic tissues.

A number of factors may influence the relationship between the drugs and gingival tissues, such as: age, genetic predisposition, pharmacokinetic variables, alteration in gingival connective tissue homeostasis, ultrastructural factors, inflammation and drug action on growth factors (11). Severity of gingival enlargement in patients taking calcium channel blockers is associated with poor plaque control (12) and is proportionate with the degree of plaque induced inflammation (7). Gender might also influence the occurrence of gingival enlargement, with males being three times more likely to develop overgrowth.

In this case, local environmental factors such as poor plaque control with heavy deposits of calculus, crowding, malocclusion and last but not least, systemic factors such as uremic milieu, have contributed to the hyperplastic response of the gingival tissues to amlodipine.

We have recommended the substitution or withdrawal of amlodipine because of the risk of recurrence of GO. But another important reason for amlodipine withdrawal is the documented detrimental effect of calcium channel blockers on cyst formation. In ADPKD, the main factor that induces cyst formation and enlargement of the kidneys, is altered proliferation of epithelial cells. Studies have shown that cyclic adenosine monophosphate (cAMP) has a mitogenic role in cystic kidneys but is antimitogenic in normal human kidneys because of the existing of a reduced steady-state level of intracellular calcium in cystic cells, compared to normal cells. Blocking of intracellular calcium intake with calcium channel blockers such as amlodipine induces cAMP-dependent cell proliferation in normal renal cells (13). However, it is the decision of the patient's physician to change or to withdraw the drug.

This case report has also demonstrated that even without withdrawal or substitution of the causative drug and with no changes in drug dosage, periodontal treatment alone can yield satisfactory clinical response, as observed also by Ikawa et al., in 2002 (14).

However, there is a possibility for the recurrence of GO, as long as the associated medication is continued and with persistency of other risk factors (15). In this case, aggravation of renal disease and of hypertension could trigger recurrence of GO and of continuing bone loss. Some studies have shown that substitution of amlodipine or nifedipine with a different calcium channel blocker, such as verapamil might be followed by a significant reduction in gingival overgrowth, but this happens more frequently when the substitution antihypertensive drug has a different chemical structure, such as ACE (16), ARBs (17-19) or thiazide diuretics (20) (21). Raman et al. 1988 (22) reported that drug withdrawal was followed by a decrease in gingival overgrowth size within one week and sometimes by full resolution.

\section{CONCLUSIONS}

If possible, all patients who are about to receive drugs which could induce gingival overgrowth, such as calcium channel blockers, should have a detailed periodontal assessment performed and if any periodontal disease discovered, treated properly. All these patients should undergo non-surgical therapy in order to at least, diminish the overgrowth to a more acceptable level, aesthetically and practically, allowing the patient to perform a good oral hygiene.

If necessary, surgery is performed, scalpel gingivectomy being the most frequently selected method. Also, substitution or withdrawal of the drug could be an option, at patient's physician choice.

Supportive follow-up is required to monitor patient's gingival and periodontal status, to assess and 
reinforce oral hygiene measures and to periodically provide professional care in order (23) to prevent recurrence of gingival enlargement.

\section{Conflict of interests}

The authors declare that there is no conflict of interests regarding the publication of this article.

\section{REFERENCES}

1. Harrap S.B., Davies D.L., Macnicol A.M. et al. Renal, cardiovascular and hormonal characteristics of young adults with autosomal dominant polycystic kidney disease. Kidney Int. 1991; 40(3),501-8

2. Ecder T., Chapman A.B., Brosnahan G.M., Edelstein C.L., Johnson A.M., Schrier R.W. Effect of antihypertensive therapy on renal function and urinary albumin excretion in hypertensive patients with autosomal dominant polycystic kidney disease. American journal of kidney diseases. 2000; 35(3),427-32

3. Seymour R., Ellis J., Thomason J., Monkman S., Idle J. Amlodipine induced gingival overgrowth. Journal of clinical periodontology. 1994; 21(4),281-3

4. Armitage G.C. Development of a classification system for periodontal diseases and conditions. Annals of periodontology 4(1), 1-6 (1999).

5. Ilgenli T., Atilla G., Baylas H. Effectiveness of periodontal therapy in patients with drug-induced gingival overgrowth. Long-term results. Journal of periodontology. 1999; 70(9), 967-72

6. Jorgensen M.G. Prevalence of Amlodipine-Related Gingival Hyperplasia*. Journal of periodontology. 1997; 68(7), 676-8

7. Ellis J.S., Seymour R.A., Steele J.G., Robertson P., Butler T.J., Thomason J.M. Prevalence of gingival overgrowth induced by calcium channel blockers: a community-based study. Journal of periodontology. 1999; 70(1),63-7

8. Ono M., Tanaka S., Takeuchi R. et al. Prevalence of amlodipineinduced gingival overgrowth. International Journal of Oral-Medical Sciences. 2010; 9(2), 96-100

9. Nery E.B., Edson R.G., Lee K.K., Pruthi V.K., Watson J. Prevalence of nifedipine-induced gingival hyperplasia. Journal of periodontology. 1995; 66(7), 572-8

10. Meraw S.J., Sheridan P.J. Medically induced gingival hyperplasia. In: Mayo Clinic Proceedings. 1998.

11. Seymour R., Thomason J., Ellis J. The pathogenesis of drug induced gingival overgrowth. Journal of clinical periodontology. 1996; 23(3), 165-75

12. Casetta I., Granieri E., Desidera M. et al. Phenytoin-induced gingival overgrowth: A community-based cross-sectional study in Ferrara, Italy. Neuroepidemiology. 1997; 16(6), 296-303
13. Nagao S., Nishii K., Yoshihara D. et al. Calcium channel inhibition accelerates polycystic kidney disease progression in the Cy/\&plus; rat.Kidney international 73(3), 269-277 (2008).

14. Ikawa K., Ikawa M., Shimauchi H., Iwakura M., Sakamoto S. Treatment of gingival overgrowth induced by manidipine administration. A case report. Journal of periodontology. 2002; 73(1), 115-22

15. Mavrogiannis M., Ellis J., Thomason J., Seymour R. The management of drug induced gingival overgrowth. Journal of clinical periodontology. 2006; 33(6), 434-9

16. Puolijoki H., Siitonen L., Saha H., Suojanen I. Gingival hyperplasia caused by nifedipine.Proceedings of the Finnish Dental Society. Suomen Hammaslääkäriseuran toimituksia.1988; 84(5-6),311

17. Mohapatra B., Kar B., Das P. Nifedipine-induced gum hyperplasia. The Journal of the Association of Physicians of India. 1991; 39(6),506

18. James J., Linden G. Nifedipine-induced gingival hyperplasia. Dental update. 1992; 19(10), 440-1

19. Aldemir N., Begenik H., Emre H., Erdur F., Soyoral Y. Amlodipineinduced gingival hyperplasia in chronic renal failure: a case report. African health sciences. 2013; 12(4), 576-8

20. Nishikawa S., Tada H., Hamasaki A. et al. Nifedipine-Induced Gingival Hyperplasia: A Clinical and In Vitro Study*.Journal of periodontology. 1991; 62(1),30-5

21. Ellis J., Seymour R., Thomason J.M., Monkman S., Idle J. Gingival sequestration of amlodipine and amlodipine-induced gingival overgrowth. The Lancet. 1993; 341(8852),1102-3

22. Raman P., Mishra V., Singh D. Nifedipine induced gingival hyperplasia. The Journal of the Association of Physicians of India 1998; 36(3), 231-3

23. Hallmon W.W., Rossmann J.A. The role of drugs in the pathogenesis of gingival overgrowth. A collective review of current concepts. Periodontology. 2000; 21(1), 176-96 